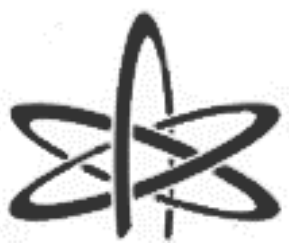

BJRS
BRAZILIAN JOURNAL

$\mathrm{OF}$

RADIATION SCIENCES

08-03B (2021) 01-08

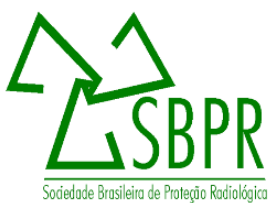

\title{
Performance of an extrapolation chamber in computed tomography standard beams
}

\author{
Maysa Costa Castro, Natállia Fiorini Silva, Linda Viola Ehlin Caldas \\ Instituto de Pesquisas Energéticas e NUcleares (IPEN/CNEN) \\ maysadecastro@gmail.com
}

\begin{abstract}
Among the medical uses of ionizing radiations, the computed tomography (CT) diagnostic exams are responsible for the highest dose values to the patients. The dosimetry procedure in CT scanner beams makes use of pencil ionization chambers with sensitive volume lengths of $10 \mathrm{~cm}$. The aim of its calibration is to compare the values that are obtained with the instrument to be calibrated and a standard reference system. However, there is no primary standard system for this kind of radiation beam. Therefore, an extrapolation ionization chamber, built at the Calibration Laboratory (LCI), was used to establish a CT primary standard. The objective of this work was to perform some characterization tests (short- and medium-term stabilities, saturation curve, polarity effect and ion collection efficiency) in the standard X-ray beams established for computed tomography at the LCI.
\end{abstract}

Keywords: Extrapolation chamber, computed tomography, dosimetry. 


\section{INTRODUCTION}

The use of Computed Tomography (CT) for diagnostic images has been growing due to technological advances of this equipment [1]. Therefore, there is a concern regarding the dose received by the patients undergoing this procedure, because it uses higher radiation doses when compared to other fields of conventional radiology, and this procedure needs to be performed with the highest precision and accuracy possible.

For the dosimetry of CT beams, the radiation detector is usually a pencil-type ionization chamber. It presents a uniform response to the incident radiation beam from all angles. However, there is no primary standard system for this kind of radiation beam. It was determined to use a homemade extrapolation chamber to establish a CT primary standard [2,3].

The extrapolation chamber is a parallel-plate ionization chamber which enables the variation of the distance between the collecting electrode and the entrance window, allowing different sensitive air volumes. This kind of ionization chamber is mostly utilized in beta radiation dosimetry [2], and it was tested for low-energy radiation beams too, showing results within the internationally acceptable limits [3-5].

This work had the objective to study the response stability of an extrapolation chamber, as well as obtaining the saturation curve, polarity effect and ion collection efficiency in standard CT beams of a calibration laboratory.

\section{MATERIALS AND METHODS}

The extrapolation chamber tested in this work has a collecting electrode of $30 \mathrm{~mm}$ in diameter, the entrance window is made of aluminized polyethylene terephthalate with density of $0.84 \mathrm{mg} / \mathrm{cm}^{2}$, and the guard ring is made of graphite; this chamber was developed at the LCI [2].

For the short and medium-term stability study of the extrapolation chamber, a ${ }^{90} \mathrm{Sr}+{ }^{90} \mathrm{Y}$ control source $(33 \mathrm{MBq})$ was utilized. Ten consecutive measurements were taken each time during a period 
of 4 months. All of the mean values were corrected for the standard environmental conditions. Figure 1 shows the experimental setup for these two tests. For the short-term stability test (diagnostic radiology qualities) of the extrapolation chamber, the standard deviation must not exceed $1 \%$. For the medium-term stability test the results obtained for the short-term stability test on different days were considered, and the standard deviation should not exceed $2 \%$ [6].

Figure 1: Experimental setup for the extrapolation chamber with the control source.

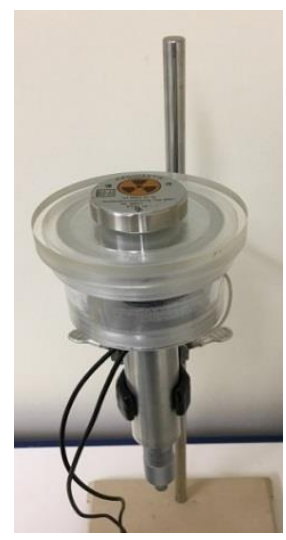

In order to obtain the saturation curve, polarity effect and ion collection efficiency of the extrapolation chamber, an X-ray system Pantak/Seifert (ISOVOLT model 160HS) was utilized, which operates up to $160 \mathrm{kV}$. Ten consecutive measurements were taken, and all of them were corrected according to the standard environmental conditions too. Table 1 presents the CT radiation qualities at the LCI, and Figure 2 shows the experimental setup when the extrapolation chamber was utilized in the X-ray system.

Table 1: Characteristics of the CT standard X radiation qualities at the LCI, based on the Report IEC 61267 [6].

\begin{tabular}{ccccc}
\hline $\begin{array}{c}\text { Radiation } \\
\text { Quality }\end{array}$ & $\begin{array}{c}\text { Tube Voltage } \\
(\mathrm{kV})\end{array}$ & $\begin{array}{c}\text { Tube Current } \\
(\mathrm{mA})\end{array}$ & $\begin{array}{c}\text { HVL } \\
(\mathrm{mmAl})\end{array}$ & $\begin{array}{c}\text { Air Kerma Rate } \\
(\mathrm{mGy} / \mathrm{min})\end{array}$ \\
\hline RQT 8 & 100 & 10 & 6.9 & 22.0 \\
RQT 9 $\dagger$ & 120 & 10 & 8.4 & 34.0 \\
RQT 10 & 150 & 10 & 10.1 & 57.0 \\
\hline
\end{tabular}

$\dagger$ Reference CT radiation quality at LCI/IPEN

HVL: Half-Value Layer 
Figure 2: Experimental setup for the extrapolation chamber in the X-ray system.

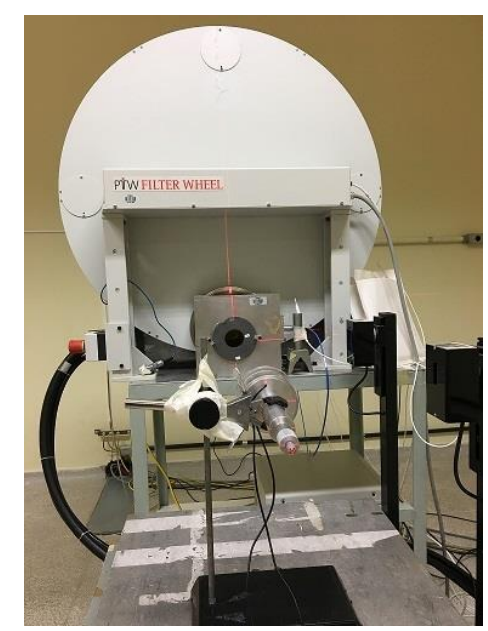

For the polarity effect test the ratio of the ionization currents obtained for positive and negative polarities must not exceed 1\%; therefore, the results should be in the range $0.99-1.01$. It was possible to calculate the ion collection efficiency of the extrapolation chamber, where the standard deviation must not exceed 95\% [6], using the following equation [7]:

$$
K_{s}=\frac{\left(V_{1} / V_{2}\right)^{2}-1}{\left(V_{1} / V_{2}\right)^{2}-\left(M_{1} / M_{2}\right)}
$$

where: $M_{1}$ and $M_{2}$ are the measures obtained with the voltages $V_{1}=+50 \mathrm{~V}$ and $V_{2}$ (for each of the other voltages applied to the extrapolation chamber), respectively.

The uncertainties of type A and type B were determined, with the combined uncertainty of a coverage factor $\mathrm{k}=2$ when necessary.

\section{RESULTS AND DISCUSSION}

The results for the short- and medium-term stability tests for the extrapolation chamber are presented. Figure 3 shows the response normalized to the mean value of the first ten measurements of the extrapolation chamber. The result for the short-term stability test was $0.88 \%$, in accordance with IEC 61267 [6]. 
Figure 3: Medium-term stability for the extrapolation chamber; the maximum uncertainty of the measurements was $0.61 \%$.

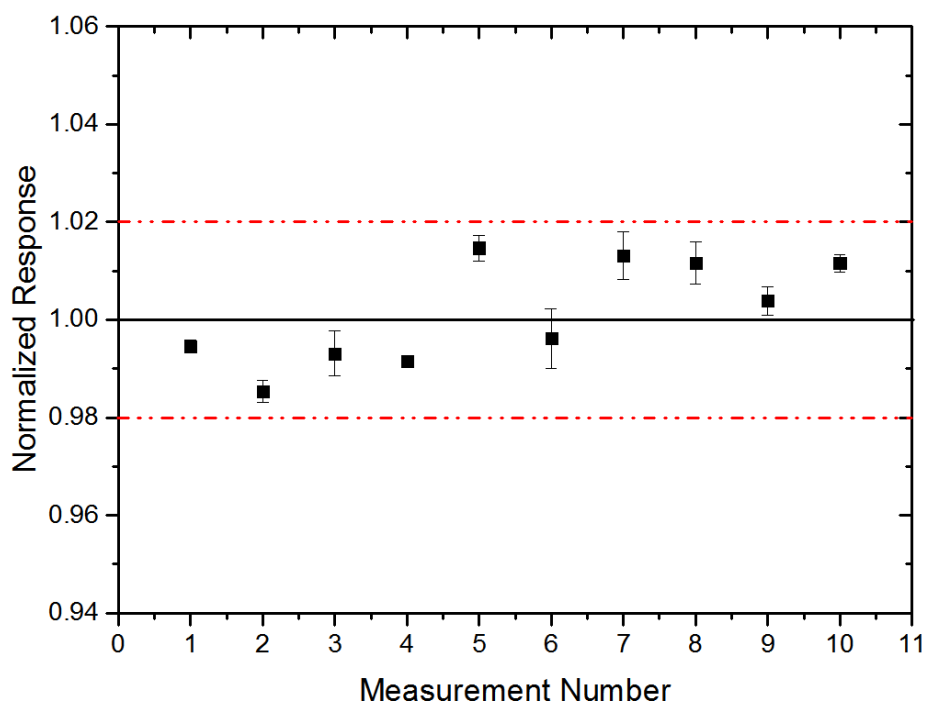

As observed in Figure 3, the medium-term stability of the extrapolation chamber is within the recommended limits [6].

The saturation curve for the extrapolation ionization chamber in the reference CT radiation quality of RQT 9 can be seen in Figure 4.

Figure 4: Saturation curve of the extrapolation chamber for the reference radiation quality (RQT 9). The maximum measurement uncertainty was $0.0003 \%$.

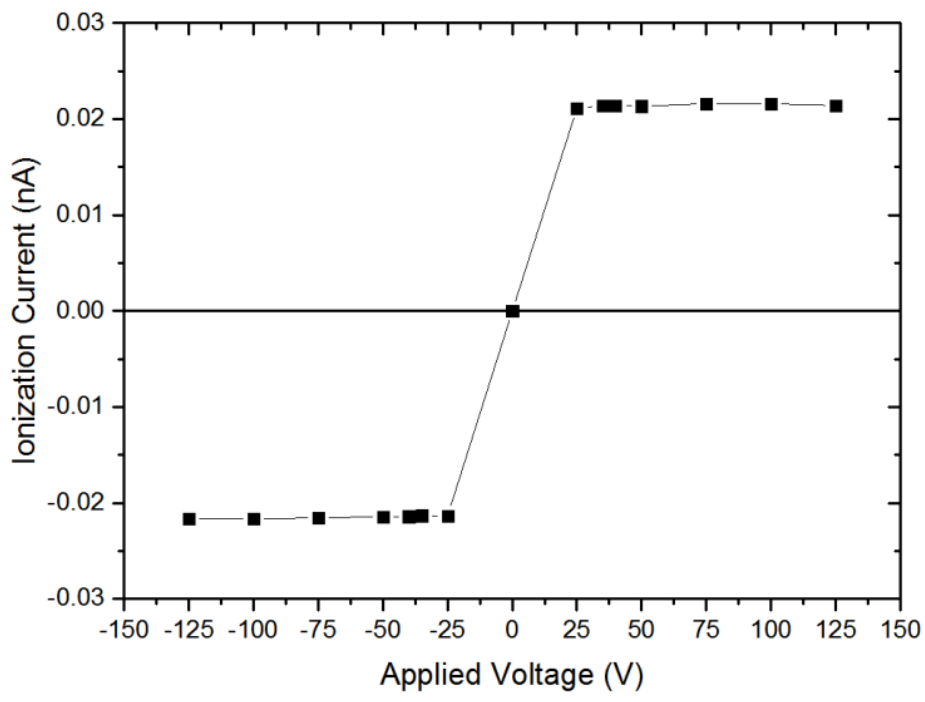


As can be observed in Figure 2, the saturation curve presents similar behavior for the positive and negative voltages. The measurements obtained for the polarity effect can show this behavior, as seen in Table 2.

Table 2: Polarity effect of the extrapolation chamber.

\begin{tabular}{cc}
\hline Voltage $(\mathrm{V})$ & Polarity Effect \\
\hline $\mathbf{2 5}$ & 0.99 \\
$\mathbf{3 5}$ & 1.00 \\
$\mathbf{4 0}$ & 1.00 \\
$\mathbf{5 0}$ & 1.00 \\
$\mathbf{7 5}$ & 1.00 \\
$\mathbf{1 0 0}$ & 1.00 \\
$\mathbf{1 2 5}$ & 0.99 \\
\hline
\end{tabular}

The polarity effect results of the extrapolation chamber are within the internationally acceptable limits [6].

The ion collection efficiency was obtained using the measurements of the saturation curve, but this test analyzes just the positive voltages. The results show a relation between ionization current and saturation current (using the voltage of $50 \mathrm{~V}$ ). The results for ion collection efficiency can be seen in Table 3.

Table 3: Ion collection efficiency of the extrapolation chamber.

\begin{tabular}{cc}
\hline $\begin{array}{c}\text { Voltage } \boldsymbol{V}_{\boldsymbol{2}} \\
(\mathbf{V})\end{array}$ & $\begin{array}{c}\text { Ion Collection } \\
\text { Efficiency (\%) }\end{array}$ \\
\hline $\mathbf{2 5}$ & 98.38 \\
$\mathbf{3 5}$ & 99.78 \\
$\mathbf{4 0}$ & 99.26 \\
$\mathbf{7 5}$ & 99.06 \\
$\mathbf{1 0 0}$ & 99.63 \\
$\mathbf{1 2 5}$ & 99.93 \\
\hline
\end{tabular}

The results obtained for the ion collection efficiency of the extrapolation chamber response are within the recommended limits [6].

\section{CONCLUSION}

The results obtained for the short- and medium-term stability tests of the homemade extrapolation chamber using the control source $\left({ }^{90} \mathrm{Sr}+{ }^{90} \mathrm{Y}\right)$ were in agreement with the international recommen- 
dations. When the extrapolation chamber was tested in the standard computed tomography beams, the results obtained for the saturation curve, polarity effect and ion collection efficiency were also within the recommended limits (IEC 61674).

\section{ACKNOWLEDGMENTS}

The authors acknowledge the partial financial support from the Brazilian agencies: CNEN, CNPq (Project 301335/2016-8) and CAPES (Project 554/2018).

\section{REFERENCES}

[1] BOONE, J. M. The trouble with CTDI100. Med. Phys. 34(4) 1364-1371, 2007.

[2] DIAS, S.K.; CALDAS, L V E. Development of an extrapolation chamber for the calibration of beta-ray applicators. IEEE Trans. Nucl. Sci. 45, 1666-1669, 1998.

[3] DIAS, S.K.; CALDAS, L V E. Extrapolation chamber response in low-energy radiation standard beams. J. Appl. Phys. 89, 669-671, 2001.

[4] NEVES, L P; SILVA, E A B; PERINI, A P; MAIADANA, N L; CALDAS, L V E. Characterization of an extrapolation chamber for low-energy X-rays: Experimental and Monte Carlo preliminary results. Appl. Radiat. Isot. 70, 1388-1391, 2012.

[5] FIGUEIREDO, M.T.T; SILVA, T.A. Determination of the effective volume of an extrapolation chamber for X-ray dosimetry, In: X CONGRESO REGIONAL LATINOAMERICANO IRPA DE PROTECTIÓN Y SEGURIDAD RADIOLÓGICA, 2015, Buenos Aires. Annals 2015.

[6] IEC - International Electrotechnical Commission. Medical Diagnostic X-ray Equipment. Radiation Conditions for Use in the Determination of Characteristics. IEC 61267. 2nd ed. Genève IEC, 2005. 
[7] IAEA - International Atomic Energy Agency. Calibration of Radiation Protection Monitoring Instruments. SRS 16, Vienna IAEA, 2001. 